

\title{
A universal formulation for indentation whatever the indenter geometry
}

Xavier Hernot, Olivier Bartier, Gerard Mauvoisin, Jean-Marc Collin

\section{To cite this version:}

Xavier Hernot, Olivier Bartier, Gerard Mauvoisin, Jean-Marc Collin. A universal formulation for indentation whatever the indenter geometry. Mechanics of Materials, 2015, 81, pp.101-109. 10.1016/j.mechmat.2014.11.006 . hal-01157486

\section{HAL Id: hal-01157486 \\ https://hal-univ-rennes1.archives-ouvertes.fr/hal-01157486}

Submitted on 28 May 2015

HAL is a multi-disciplinary open access archive for the deposit and dissemination of scientific research documents, whether they are published or not. The documents may come from teaching and research institutions in France or abroad, or from public or private research centers.
L'archive ouverte pluridisciplinaire HAL, est destinée au dépôt et à la diffusion de documents scientifiques de niveau recherche, publiés ou non, émanant des établissements d'enseignement et de recherche français ou étrangers, des laboratoires publics ou privés. 


\title{
A universal formulation for indentation whatever the indenter geometry
}

\author{
X. Hernot ${ }^{\mathrm{a}}$, O. Bartier ${ }^{\mathrm{a}}$, G. Mauvoisin ${ }^{\mathrm{a}}$, J.-M. Collin ${ }^{\mathrm{a}, \mathrm{b}}$ \\ ${ }^{a}$ LGCGM, EA 3913, 20 Avenue des Buttes de Coësmes, 35708 Rennes Cedex 7, France \\ ${ }^{\mathrm{b}}$ Université de Nantes, 1 quai de Tourville, BP 13522, 44035 Nantes Cedex 1, France
}

\begin{abstract}
We propose in this paper a geometrical equivalence between a shape described by a power law and a conical geometry. A theoretical and numerical study has allowed us to generalize an equivalence between the conical geometrical parameter $\tan \beta$ and the spherical or power law shape geometrical parameter $a_{d} / R$. Moreover, in order to superpose indentation data whatever the geometry, a new pile-up parameter $\Delta$ has been introduced. For one set of mechanical properties of the tested sample, this new formulation leads to a perfect superposition between conical, power law shape and spherical indentation data. At the end of this paper, we propose a comparison between the results proposed in literature and the present formulation.
\end{abstract}

\section{Introduction}

The indentation test was developed several decades ago in order to determine certain mechanical properties. It becomes an important subject of interest for many mechanical engineering industries. An instrumented indentation test consists in measuring simultaneously indentation load $F$ and indentation depth $h$ during the penetration of an indenter into a sample. The contact hardness $H$ (Meyer, 1908) has been introduced by the following relationship:

$H=\frac{F}{\pi a_{c}^{2}}$

In Eq. (1) contact radius $a_{c}$ is a fundamental indentation data, it is the link between indenter and tested sample. Different kind of indenter geometries are commonly used, they can be grouped in two families:

- the sharp indenters (Vickers, Berkovich, Knoop and cone) which can be correctly modelled by an equivalent conical geometry, they can be called axisymmetric cones. For Vickers and Berkovich indenters, the half-apex angle of the equivalent cone is $\theta=70.3^{\circ}$. In the theoretical studies formulations are often written with $\beta=\pi / 2-\theta$.

- the spherical indenters (radius $R$ ) which can be correctly modelled by a parabolic geometry for the low $a_{c} / R$ ratios.

For conical indentation, it is commonly assumed that the indentation pressure on elasticperfectly plastic materials may be correlated on a non-dimensional graph of $H / Y$ as a function of the so called Johnson parameter $E^{*} \tan \beta / Y$, where $E^{*}$ is the reduced modulus of the contact (Johnson, 1970, Larsson, 2008, Bartier and Hernot, 2012). For a conical indenter with a fixed $\beta, H / Y$ is a constant during indentation test while it varies with the $a_{c} / R$ ratio for parabolic or spherical indenters. For spherical indention, it is considered that $\tan \beta \approx \sin \beta=a_{c} / R$ where $\beta$ is the contact angle between the indenter and the tested sample at the edge of the contact. 
Using the equality given above, Johnson (1970) found a good correlation between the indentation pressures obtained for conical and spherical indentation.

Because surface's sample can present sink-in or pile-up, it is not possible to directly determine the contact radius $a_{c}$ from the indentation depth $h$. A great number of studies have been conducted in order to quantify sink-in or pile-up degree. The ratio between the contact depth $h_{c}$ and the indentation depth $h$ is called $c^{k} . c^{1}$ or $c^{2}$ are used respectively for conical or spherical indenter. Using these ratios, the contact radius can be calculated with Eq. (2) and Eq. (3) respectively for conical and spherical indenter:

$$
\begin{aligned}
& a_{c}=\frac{c^{1}}{\tan \beta} h \\
& a_{c}^{2}=2 R c^{2} h
\end{aligned}
$$

Eq. (3) is often used in the literature; it is based on an approximation of the exact function of a sphere (Eq. (4)) by a parabolic geometry. In this approximation, $h_{c}{ }^{2}$ is considered as negligible compared to $2 R h_{c}$.

$a_{c}^{2}=2 R h_{c}-h_{c}^{2}$

This is the reason why the pile-up degree has been quantified by $c^{2}$ in the case of spherical indentation. Many authors have developed models to quantify $c^{2}$ depending on the mechanical properties of the tested sample. At the beginning, this parameter has been considered as a constant during a spherical indentation test. It is with this assumption that Matthews (1980), Hill et al. (1989), Biwa and Storakers (1995), Taljat et al. (1998), Alcala et al. (2000) showed that $c^{2}$ is linked to the strain hardening exponent of the indented material. It has been shown that the assumption of constant $\mathrm{c}^{2}$ is only valid for rigid plastic materials and low values of contact radius (Bartier and Hernot, 2012). In the case of elastic-plastic materials, $c^{2}$ depends not only on the strain hardening exponent, $n$, but also on the relative amount of elastic and plastic deformation as characterized by the non-dimensional material parameter $E / Y$ and the non-dimensional depth of penetration, $h / R$,(Taljat and Pharr, 2004, Lee et al. 2005, Hernot et al., 2006, Kim et al, 2006, Bartier et al., 2010).

For an elastic-plastic material, it is thus now well known that the pile-up degree changes during a spherical indentation test while it remains constant during a conical indentation test. Concerning the conical indentation, the pile-up degree does not change during the indentation test because of the self-similarity of the geometry.

Table. 1. shows the comparison between $c^{1}$ and $c^{2}$ calculated from two different formulations (Bower et al., 1993 and Felder, 2006) for a rigid perfectly plastic sample. This table illustrates that even if the mechanical properties of the sample are the same, the pile-up parameters have not the same values for conical or spherical geometries.

\begin{tabular}{llll}
\hline $\mathrm{p}$ & 1 (cone) & 2 (sphere) & \\
\cline { 2 - 3 } & $c$ & $c^{2}$ & $H^{*}=H / \sigma_{0}$ \\
\hline Zero friction & 1.263 & 1.44 & 3.05 \\
\hline Sticking friction & 1.209 & 1.334 & 3.21 \\
\hline Model $(s=4.35)$ & 1.219 & 1.37 & 3.62 \\
\hline
\end{tabular}

Table. 1. Comparison between $C^{l}$ and $C^{2}$ (Felder, 2006) 
To conclude, the problem of elastic solids indented by punches of various profiles was solved by Love ((1939), Segedin (1957) and Sneddon (1965). For elastic indentation, the sinkin/pile-up degree remains constant and correlations between conical, spherical or punch of arbitrary profile can be easy deduced from analytical formulations. In the case of indentation of elastic-plastic solids, it has been shown that the pile-up degree remains constant during a conical indentation while it changes during a spherical indentation. Indeed, for the conical indentation, the contact radius is linked to the pile-up depth with a constant geometrical parameter $\tan \beta$. Concerning the spherical indentation, the pile-up degree increases with the $a_{c} / R$ ratio. However, it does not yet exist a formulation to make the link between $c^{2}$ and $c^{l}$. The aim of this work is to propose a universal normalized formulation in order to superpose the results obtained in indentation whatever the geometry when plasticity occurs.

\section{Indentation morphology for conical indentation and indentation with an indenter with a shape described by a power law}

It has been shown, in section 1 , that using $c^{2}$ for spherical indentation come from an approximation of a sphere by a parabolic geometry when the $a_{d} / R$ ratio is low. However, $c^{2}$ is valid for a whole indentation test realized with a parabolic indenter. We propose to begin with the comparison between an indenter shape described by a power law (Eq. (5) with $k>1$ ) and a conical indenter (Eq. (6)). In the case where $\mathrm{k}=2, \mathrm{R}$ is the radius of the osculating circle at the bottom of the indenter. For $\mathrm{k}>2, \mathrm{R}$ is introduced to keep a length parameter in equation (6).

$$
\begin{aligned}
& y=\frac{r^{k}}{(2 R)^{k-1}} \\
& y=\tan \beta . r
\end{aligned}
$$

In Eqs (5) and (6) $r$ and $y$ are respectively the horizontal and vertical coordinate. In Eq. (5) $k$ is the exponent of the power geometry and $R$ is introduced in order to make an analogy with Eq. (3). We can notice that considering $\mathrm{k}=1$ leads to the description of an axisymmetric cone with an angle $\beta=45^{\circ}$.

Fig. 1 shows the fundamental indentation data that will be used in order to develop the theoretical formulations: the contact radius $a_{c}$, the displaced volume $V$ under the contact area, the applied load $F$, the contact depth $h_{c}$ and the indentation depth $h$. 


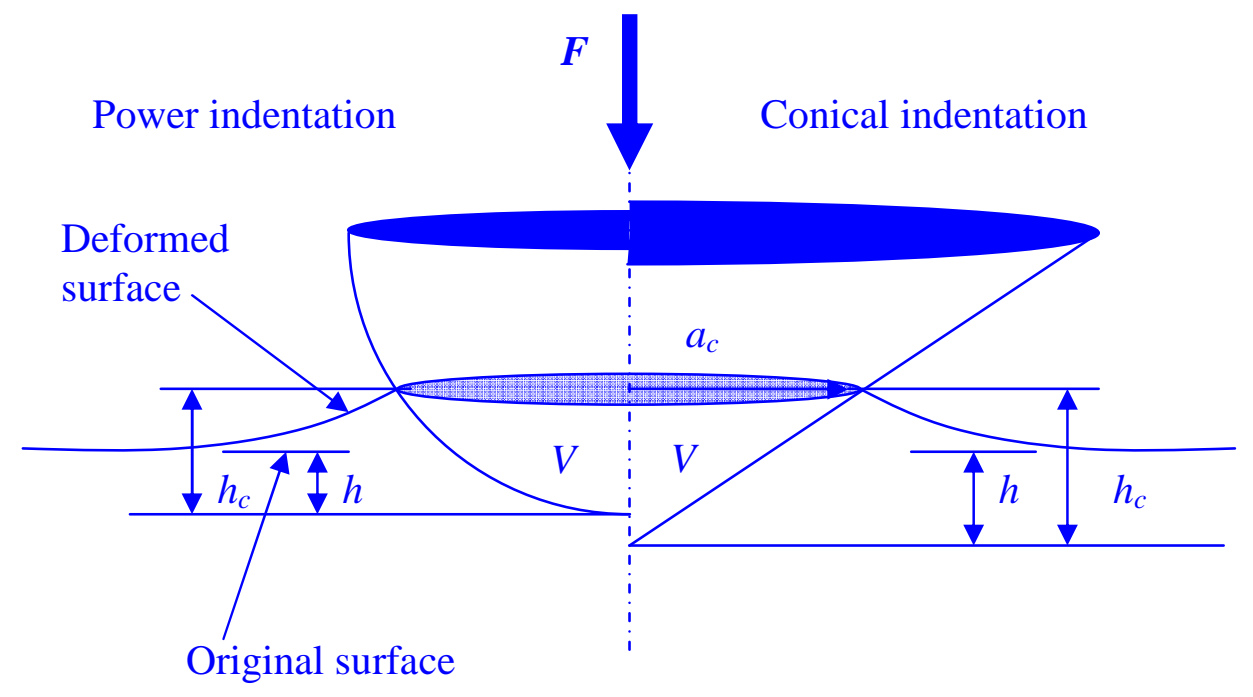

Fig. 1. Indentation data for the two indenters geometries

In the following sections, theoretical formulations will be illustrated with the results obtained by finite element simulations of indentation performed with a parabolic indenter $(k=2)$, an indenter with a shape described by a power law of $k=3$ and a conical indenter on a sample with the same mechanical properties. Indenter is considered as rigid and the contact problem is considered as frictionless. This sample is considered as elastic - plastic with isotropic hardening described by a power law associated to the $J 2$ flow stress theory. The behaviour law considered is $\sigma=E \varepsilon$ for $\sigma<Y$ and $\sigma=\sigma_{y}^{1-n} E^{n} \varepsilon^{n}$ for $\sigma>Y$. Materials properties are Young modulus $E=210 G P a$, Poisson ratio $v=0.3$, yield stress $Y=210 M P a$ and work hardening exponent $n=0.25$."

\section{Correlation between the indentation pressures $\mathrm{H} / \mathrm{Y}$}

The normalized hardness $H / Y$ obtained for conical geometries and axisymmetric geometries with a power law profile are compared. For this comparison, the so called Johnson parameter (Johnson, 1970) is reduced to $\tan \beta$ for conical indentation and $a_{\mathcal{C}} / R$ for spherical indentation. Using the same assumption for power-law shaped indenters we obtain $k\left(a_{c} / 2 R\right)^{k-1}$ which is the tangent at the edge of the contact between indenter and material. Fig. 2 shows that the curves $H / Y=f\left(k\left(a_{c} / 2 R\right)^{k-1}\right)$ for indenters with a power law profile and $H / Y=f(\tan \beta)$ for conical indenters are not superposed. This result demonstrates that the indentation pressures by axi-symetrical indenters of arbitrary profiles into elastic-plastic materials do not correlate when using the so called Johnson parameters $E^{*} \tan \beta / Y$ and $\left.E^{*}\left(k\left(a_{c} / 2 R\right)^{k-1}\right) / Y\right)$. 


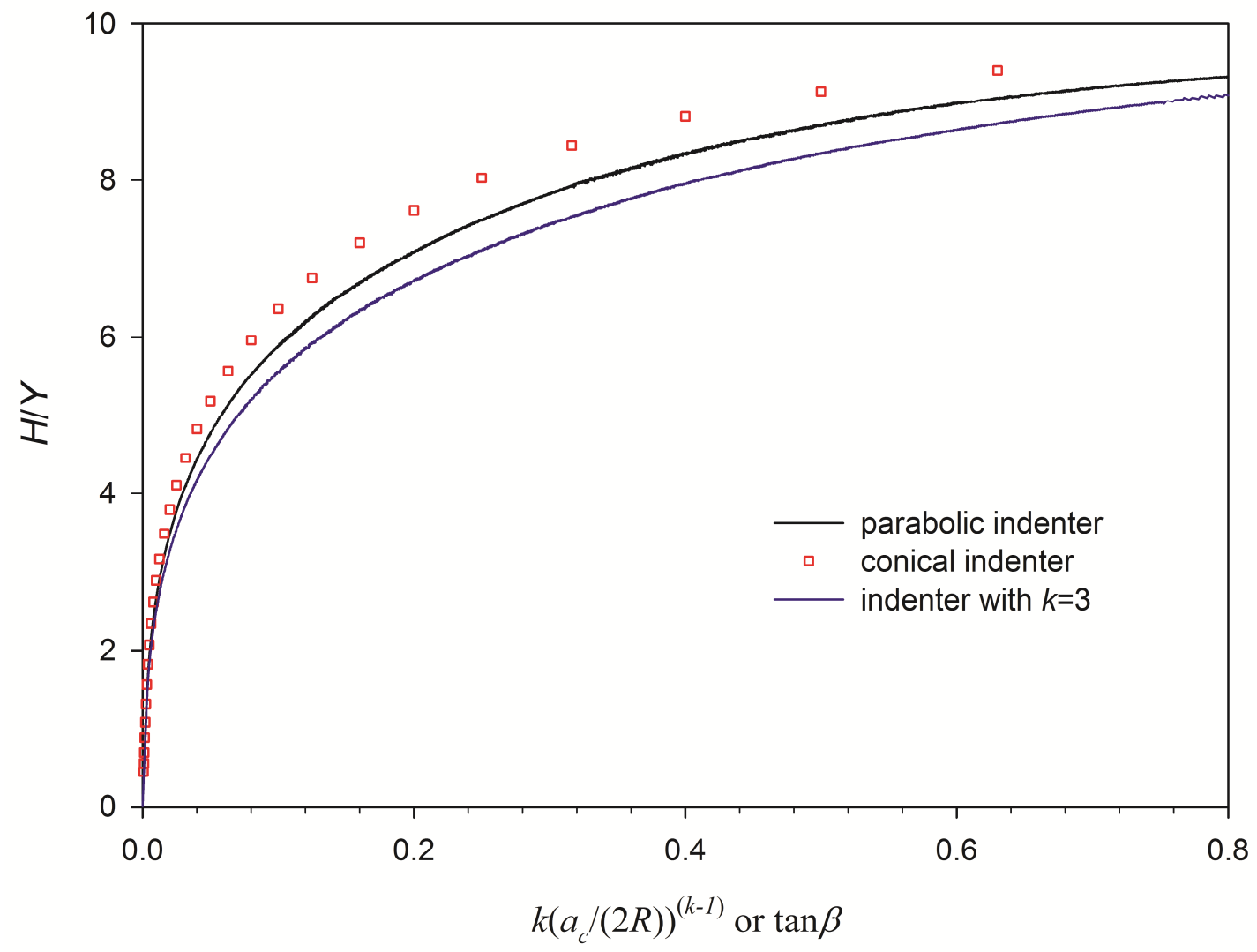

Fig. 2: Normalized hardness function of $k\left(a_{c} / 2 R\right)^{k-1}$ or $\tan \beta$

We propose to use the assumption of equality of volume displaced $V$ under the contact area for the same contact radius $a_{c}$ in order to correlate the variation of $H / Y$ obtained for various shapes of indenter. This assumption is quite similar to the one used in order to determine the equivalent cone of a Berkovich or Vickers indenter.

Using Eqs. (5) and (6) the volume of the indenter under the contact depth is given by Eq. (7) for an indenter shape described by a power law and Eq. (8) for a conical indenter:

$$
\begin{aligned}
& V=\frac{k \cdot \pi \cdot a_{c}{ }^{k+2}}{(k+2) \cdot(2 R)^{k-1}} \\
& V=\frac{\pi}{3} a_{c}^{3} \tan \beta
\end{aligned}
$$

Equality of Eqs. (7) and (8) leads to the geometrical equivalence between a shape described by a power law and a conical geometry as following:

$$
\frac{3 k}{k+2}\left(\frac{a_{c}}{2 R}\right)^{k-1}=\tan \beta
$$

Considering a parabolic indenter (with $k=2$ ) in Eq. (9) leads to the following equivalence: 
$\frac{3}{4} \frac{a_{c}}{R}=\tan \beta$

Fig. 3 shows that using formulation (9) allows the superposition of the $H / Y$ curves for geometries with a power law profile $(k=2$ and $k=3)$ and conical geometries. It is thus shown that a unique indentation test with an indenter with a shape described by a power law leads to the same hardness changes than several conical indentations. Eq. (9) gives an equivalent value of $\tan \beta$ for an indenter with a shape described by a power law.

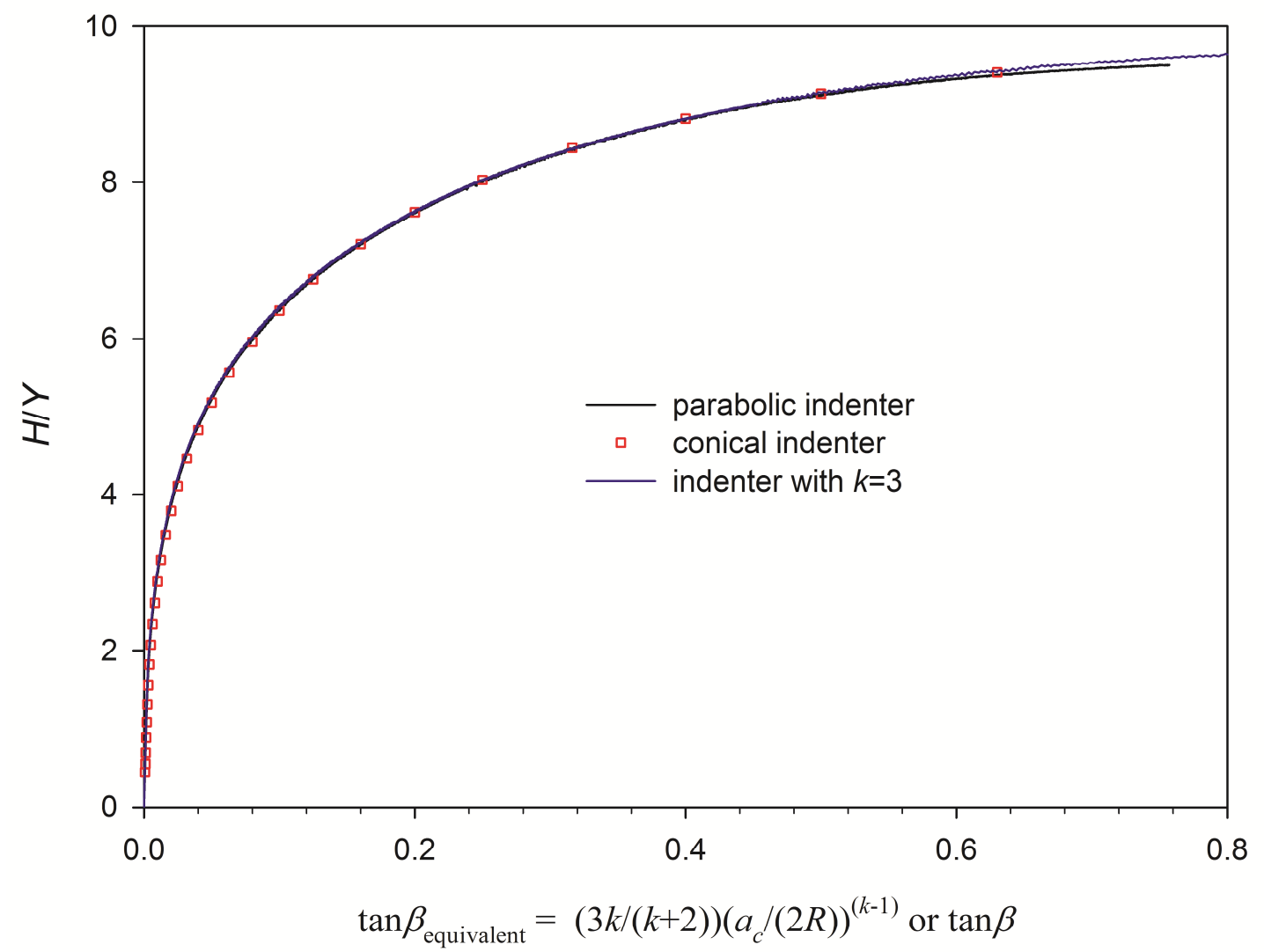

Fig. 3: Normalized hardness function of $\tan \beta_{\text {equivalent }}$

\section{Correlation between the $c^{k}=h_{c} / h$ surface deformation parameters.}

By using the transformation method suggested by Hill et al. (1989), it is shown that the indentation problem can be reduced to a form which is independent of the geometry of the punch and depends only on the material properties. By noticing that the velocity fields under a punch have certain self similar properties, it is possible to calculate the stress and the velocity field under a punch with a profile given by (5) and (6). The indentation analysis is reduced to calculating stresses and displacements in a solid, indented to a unit depth by a rigid flat punch of a unit radius. In self similarity methods $c^{k}$ was defined as an eigenvalue of the indentation. This theory has been analytically developed for linear elastic materials. However, this theory has extended to plastic and creeping materials by considering non linear elastic behaviour. (Hill et al. 1989, Storacker and Larsson 1994, Shu and Fleck 1998, Larsson 2008) and for rigid plastic materials by Biwa and Storacker (1995). From a physical point of vue, $c$ 
determines the ratio of the true to nominal contact radius under the punch, i.e $a_{d} / a . c^{k}$ corresponds to the ratio between the contact depth $h_{c}$ and the indentation depth $h$. i.e.:

$c^{k}=\frac{h_{c}}{h}$

$c$ and $c^{k}$ control the vertical displacement of the surface at the edge of the contact area. If $c$ or $c^{k}<1$, material is pushed into the surface at the edge of the contact, while if $c$ or $c^{k}>1$, material piles up at the side of the indentation. Comparison between values of $c$ or $c^{k}$ obtained for conical and spherical indentation of rigid perfectly plastic materials was performed in the literature (Bower, Felder). The examination of the values of $c$ and $c^{k}$ found by Bower and Felder, respectively shows that no identical values of $c^{k}$ is obtained for spherical and conical indentation of rigid perfectly plastic material. Fig. 4 confirms that the ratios between the contact depth and the indentation depth obtained for various shapes of indenter does not superpose on a non-dimensional graph of $c^{k}$ as a function of the so called Johnson parameter $\tan \beta$ or $k\left(a_{c} / 2 R\right)^{k-1}$. Fig. 5 also illustrates that the use of Eq. (9) leads to closer results but is not sufficient to superpose the indentation results concerning the pile-up degree. We thus can conclude that $h d h$ ratio is not a universal data to quantify the pile-up degree whatever the geometry.



Fig. 4: $h_{c} / h$ function of $k\left(a_{c} / 2 R\right)^{k-1}$ or $\tan \beta$ 




Fig. 5: $h_{c} / h$ function of $\tan \beta_{\text {equivalent }}$

\section{Definition of a new pile-up function}

\subsection{Cone and indenter with a shape described by a power law}

We propose to compare the pile-up height $h_{c}-h$ obtained for conical indentation and that obtained with an indenter described by power law profile.

Using Eqs. (5) and (11) lead to the pile-up height $h_{c}$ - $h$ for indenter with a shape described by a power law:

$$
h_{c}-h=\frac{a_{c}^{k}}{(2 R)^{k-1}}\left(1-\frac{1}{c^{k}}\right)
$$

Eqs. (6) and (11) lead to the pile-up height $h_{c}$ - $h$ for a conical indenter:

$$
h_{c}-h=\tan \beta \cdot a_{c}\left(1-\frac{1}{c^{1}}\right)
$$

When the equality of pile-up height whatever the geometry is respected, we can write: 
$h_{c}-h=\frac{a_{c}^{k}}{(2 R)^{k-1}}\left(1-\frac{1}{c^{k}}\right)=\tan \beta \cdot a_{c}\left(1-\frac{1}{c^{1}}\right)$

Using the geometrical equivalence given by Eq. (9), this equality becomes:

$\Delta=\frac{k+2}{3 k}\left(1-\frac{1}{c^{k}}\right)=\left(1-\frac{1}{c^{1}}\right)$

In this equation, a new function $\Delta$ for the pile-up height is introduced. Equality of $\Delta$ for various indentation tests indicates that $h_{c}$-h is equal when the equality of the volume $V$ displaced under the contact area for the same contact radius $a_{c}$ is respected. Considering a parabolic indenter $(k=2)$, Eq. (15) leads to the following equivalence:

$$
\Delta=\frac{2}{3}\left(1-\frac{1}{c^{2}}\right)=\left(1-\frac{1}{c^{1}}\right)
$$

We propose to compare the new pile-up function $\Delta$ given by Eq. (15) obtained for parabolic and conical geometries calculated for the same tested sample $(E / Y=1000$ and $n=0.25)$. For a conical indenter with a fixed $\beta, \Delta$ is a constant during indentation test while it increases with the $a_{c} / R$ ratio for an indenter described by a power law geometry. Fig. 6 shows that using formulation (16) allows the superposition of the $\Delta$-tan $\beta_{\text {equivalen }} t$ curves obtained with conical geometries and geometries with a power law profile while the results are not superposed when using the $h d h$ ratio (Fig. 5).

It is thus shown that a unique indentation test performed with an axisymmetric geometry with a power law profile describes the same $H / Y$ and $\Delta$ changes than several conical indenters (Fig. 3 and 6). Eq. (9) gives an equivalent value of $\tan \beta$ and Eq. (15) gives a universal pile-up function for an indenter with a shape described by a power law.

This new universal pile-up function leads to a transition between sink-in and pile-up at $\Delta=0$ whatever the mechanical properties if we have a material exhibiting a transition between sinkin and pile-up during indentation. 


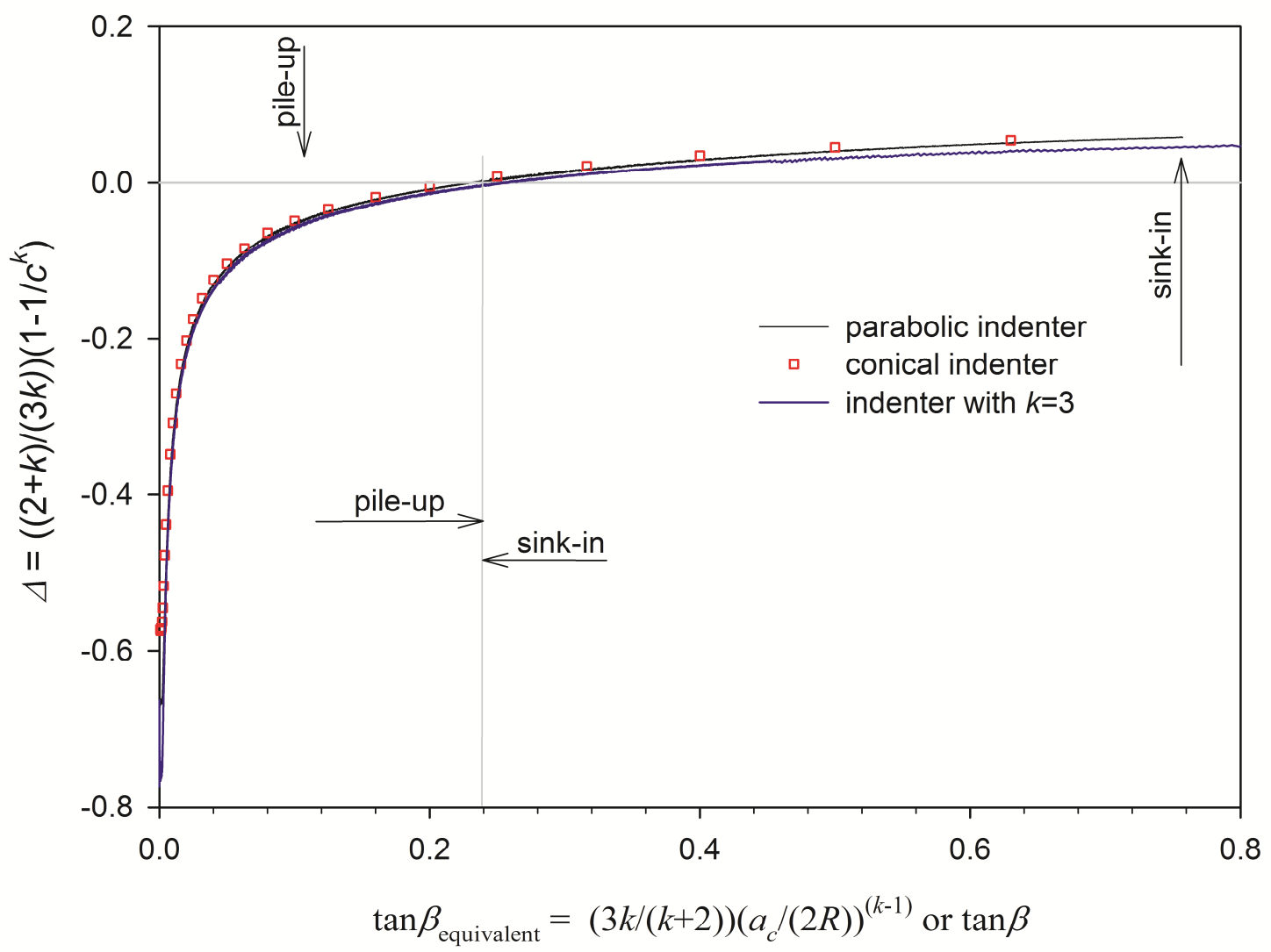

Fig. 6: New pile-up function $\Delta$ versus $\tan \beta_{\text {equivalent }}$

\subsection{Spherical and parabolic indenters}

The study of parabolic indentation was useful in order to distinguish the influence of the material and the geometrical properties on the relationship between the contact radius and the penetration depth. However, the study of spherical indentation is necessary because the ball indenter is a precision instrument which is easy to make, yet robust, inexpensive and thus often used for experimental tests. The problem for elastic solids in spherical indentation was solved by Segedin (1957) and then it was discussed by Sneddon (1965). Moreover, using a similarity approach for non linear elastic (isotropic or anisotropic) materials, Borodich (1993) has developed a theory involving indenter shapes of degree $k$. He also superposed results obtained with different spherical indenters with several radii. Despite the fact that the similarity approach proposed by Borodich is valid for elastic materials, the proposed formulations lead to a formula similar to the empirical law of Meyer. This method can be extended to other indenters shapes changing factor $k$. We can also quote Borodich and Keer (2004) and Borodich et al. (2003).

If we call $c^{s}$ the spherical $h_{c} / h$ ratio which is different to $c^{2}$ calculated for a parabolic indenter. The contact radius for spherical indenter with a radius $R$ is given by:

$a_{c}=\left(2 R h c^{s}-\left(c^{s} h\right)^{2}\right)^{0.5}$ 
For a spherical geometry, the relationship between horizontal and vertical coordinate $r$ and $y$ is:

$$
y=R-\sqrt{R^{2}-r^{2}}
$$

The volume $V$ under the contact area and the pile-up height $h_{c}-h$ are then given by the following relationships:

$$
\begin{aligned}
& V=2 \pi\left(\frac{1}{2} R \cdot a_{c}^{2}+\frac{1}{3}\left(R^{2}-a_{c}^{2}\right)^{3 / 2}-\frac{1}{3} R^{3}\right) \\
& h_{c}-h=\left(R-\sqrt{R^{2}-a_{c}^{2}}\right)\left(1-\frac{1}{c^{s}}\right)
\end{aligned}
$$

Using Eqs. (8) and (19), it is possible to determine the geometrical equivalence between spherical and conical geometry, $\tan \beta_{\text {equivalent }}$, and the new pile-up function, $\Delta$, as following:

$$
\tan \beta_{\text {equivalent }}=3 \frac{R}{a_{c}}+2\left[\left(\frac{R}{a_{c}}\right)^{2}-1\right]^{3 / 2}-2\left(\frac{R}{a_{c}}\right)^{3}=\tan \beta
$$

$\Delta=\frac{\frac{R}{a_{c}}-\sqrt{\left(\frac{R}{a_{c}}\right)^{2}-1}}{\left(3 \frac{R}{a_{c}}+2\left[\left(\frac{R}{a_{c}}\right)^{2}-1\right]^{3 / 2}-2\left(\frac{R}{a_{c}}\right)^{3}\right)}\left(1-\frac{1}{c^{s}}\right)=\left(1-\frac{1}{c^{1}}\right)$

Using Taylor series to develop Eq. (21) leads to the following approximation of tan $\beta_{\text {equivalent }}$ : $\tan \beta_{\text {equivalent }} \approx \frac{3}{4} \frac{a_{c}}{R}+\frac{1}{8}\left(\frac{a_{c}}{R}\right)^{3}+\frac{3}{64}\left(\frac{a_{c}}{R}\right)^{5}+\frac{3}{128}\left(\frac{a_{c}}{R}\right)^{7}+\frac{7}{512}\left(\frac{a_{c}}{R}\right)^{9}+O\left[\left(\frac{a_{c}}{R}\right)^{11}\right]$

In a first order approximation, we find the same formulation than the one developed for parabolic indenter $(k=2)$ in Eq. (10):

$\tan \beta_{\text {equivalent }}=\frac{3}{4} \frac{a_{c}}{R}$

In the literature, the contact radius for spherical indentation is often determined with the approximation with a parabolic geometry given by Eq. (3). (Hernot et al (2006) and Bartier and Hernot (2012)) showed that this approximation does not lead to a good prediction of the contact radius of the sphere when $a_{d} / R>0.2$ because $c^{2}$ underestimates the contact radius. When the profile of the spherical indenter cannot be approximated by a paraboloid of revolution, i.e. when $a_{d} / R>0.2$, it is necessary to use $c^{s}=h_{c} / h$ for spherical indenter in order to quantify the pile-up degree (eq. (17)). 
Fig. 7 shows that $c^{s}=h_{d} / h$ for spherical indenter and $c^{2}=h_{d} / h$ for parabolic indenter are very close until values of $a_{c} / R$ approximately equal to 0.3 . From this value, the difference becomes significant because of the difference between the shapes of the parabolic and spherical indenters. Using the geometrical equivalence between spherical and parabolic geometries defined by Eqs. (10) and (21), the new pile-up function $\Delta$ given by Eq. (22) for spherical geometry and that given by Eq. (16) for parabolic geometry are very close until large values of contact radius (Fig. 8). These result confirms that the pile-up height, $h_{c}-h$, is the same for various geometries of indenter when the equality of the volume $V$ displaced under the contact area for the same contact radius $a_{c}$ is respected.

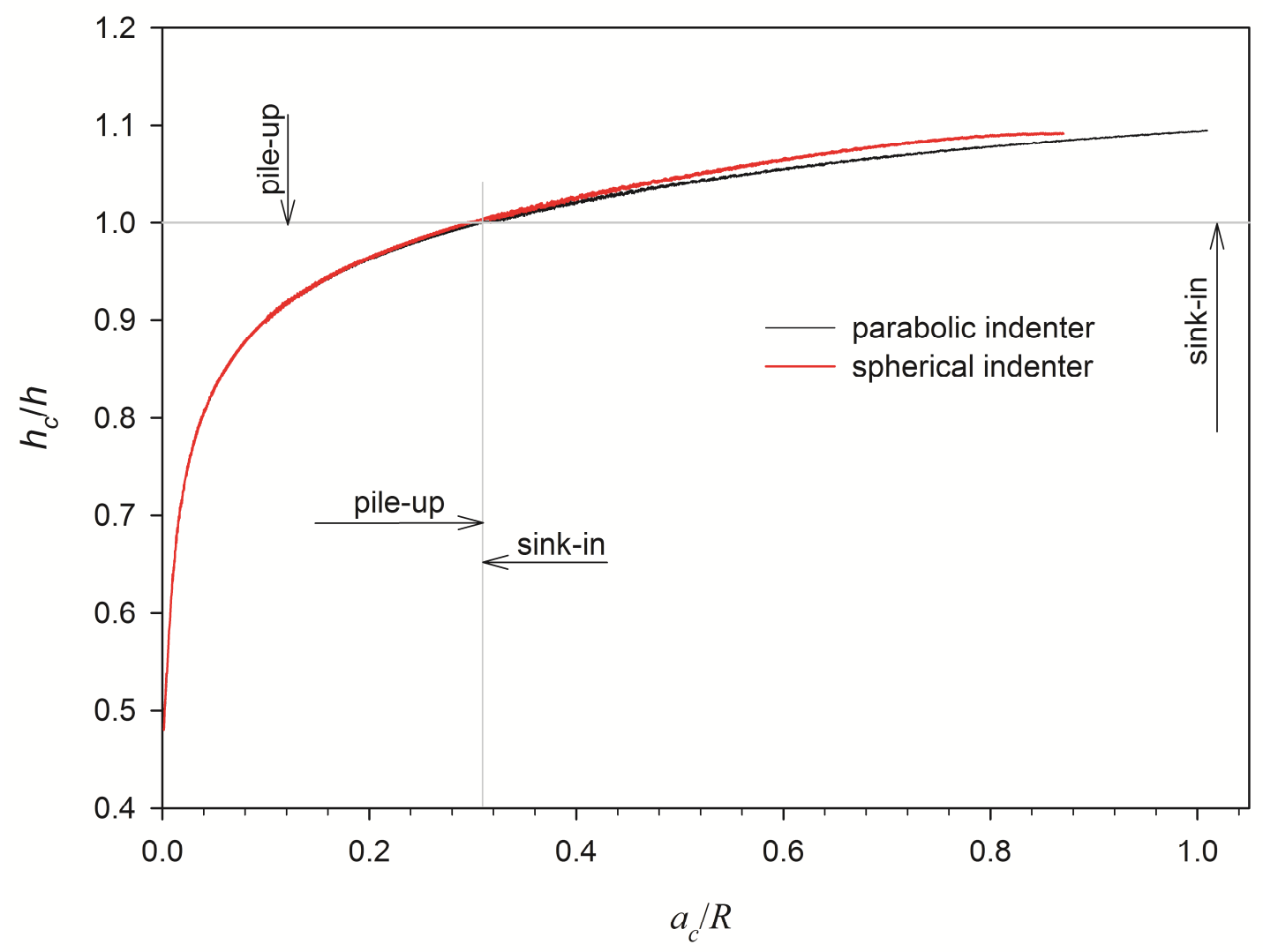

Fig. 7: Comparison between $c^{s}=h_{c} / h$ for spherical indenter and $c^{2}=h_{c} / h$ for parabolic indenter. 


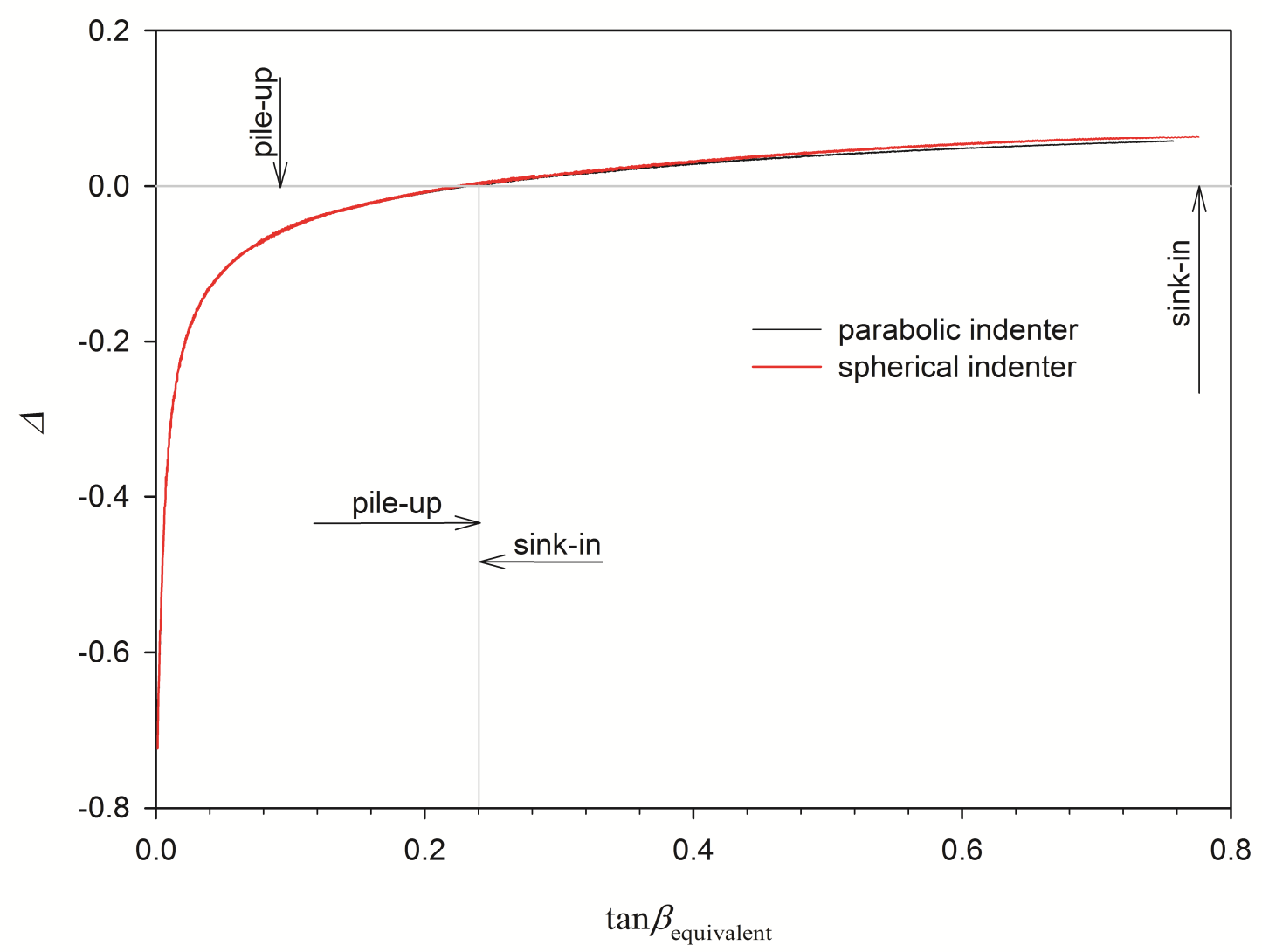

Fig. 8: New pile-up function $\Delta$ versus equivalent $\tan \beta$ for spherical and parabolic indenters.

\subsection{Comparison with literature}

In order to validate the proposed formulations, we propose to make a comparison with the literature values given by Bower et al. (1993) and Felder (2006). The literature values of $c^{1}$ and $c^{2}$ for a rigid perfectly plastic sample are given in Table 1. Bower et al. (1993) proposed different values of the $h_{c} / h$ ratio for conical and spherical indentations with or without friction. Felder (2006) proposed a unique average value of $h_{c} / h$ ratio for conical and spherical indentations. Watching the first line of Table 1., Bower et al. calculated $c^{1}=1.263$ for a frictionless conical indentation $\left(\theta=70.3^{\circ}\right)$ and $c^{2}=1.44$ for a frictionless spherical indentation which represents a difference of $14 \%$ between the two $h_{c} / h$ ratios.

Table 2. shows the values of the new pile-up parameter $\Delta$ calculated from the values of $c^{1}$ and $c^{2}$ presented in Table 1. For the Bower et al. frictionless results (first line in Table 2.), using Eq. (15) leads to $\Delta=0.208$ for the conical indenter and $\Delta=0.203$ for a parabolic indenter which represents a difference of $2.4 \%$. Using Eq. (22) leads to $\Delta=0.208\left(a_{c} / R=0.5\right)$ for spherical indenter which demonstrates a perfect superposition between the new conical and spherical pile-up parameter $\Delta$.

\begin{tabular}{|c|c|c|c|}
\hline & Conical indenter $k=1$ & Parabolic indenter $k=2$ & Sperical indenter \\
\hline Zero friction (Bower et al., 1993) & 0.208 & 0.203 & 0.208 \\
\hline Sticking friction (Bower et al., 1993) & 0.173 & 0.167 & 0.170 \\
\hline Felder model (2006) & 0.180 & 0.180 & 0.184 \\
\hline
\end{tabular}

Table 2. New pile-up parameter $\Delta$ calculated from literature values 
Concerning the three different lines in Table 2. we can see that we have very close values of $\Delta$ whatever the geometry. The differences which occur in the three different columns are due to the friction conditions. We can also see that the Felder values are more close to the sticking friction values obtained by Bower et al. than the frictionless values.

\section{Conclusion}

Geometrical assumptions have allowed us to propose a new universal formulation to superpose indentation results whatever the geometry.

First of all, a geometrical equivalence between a shape described by a power law and a conical geometry has been introduced. A comparison between spherical indenter and a shape described by a power law has allowed us to generalize the equivalence between the conical geometrical parameter $\tan \beta$ and the spherical or power law shape geometrical parameter $a_{c} / R$.

Moreover, a new pile-up parameter $\Delta$ has been introduced. It allows quantifying the pile-up degree with a same value for conical indenter, indenter with a shape described by a power law or spherical indenter while the former parameters were dependant on the indenter geometry.

Using this new formulation leads to a perfect superposition between conical, shape described by a power law and spherical indentation data. It is thus shown that a unique spherical indentation test will give the same results than several conical indentation tests (with different apex angles). The results proposed in this paper lead to good results for quite low Y/E values and high work hardening exponents for which the indentation regime can be considered as elastic-plastic. Further investigations will concern other materials for which the indentation regime will be elastic or fully-plastic.

\section{References}

Alcala J., Barone A.C., and Anglada M., 2000. The influence of plastic hardening on surface deformation modes around Vickers and spherical indents. Acta Mater. 48, 3451-3464.

Bartier O Hernot X. 2012. Phenomenological study of parabolic and spherical indentation of elastic-ideally plastic material. International Journal of Solids and Structures. 49, 2015-2026.

Bartier O., Hernot X., Mauvoisin G.. 2010. Theoretical and experimental analysis of contact radius for spherical indentation. Mechanics of Materials 42, 640-656.

Biwa S. and Storackers B., 1995. An analysis of fully plastic Brine11 indentation. J. Mech. Phys. Solids, Vol. 43, No. 8, 1303-1333.

Borodich, F.-M., 1993. The Hertz frictional contact between nonlinear elastic anisotropic bodies (the similarity approach). Int. J. S. Str., 30, 1513-1526.

Borodich F. M., Keer L. M. and Korach C.S. 2003. Analytical study of fundamental nanoindentation test relations for indenters of non-ideal shapes. Nanotechnology, 14, 7, 803.

Borodich F. M., Keer L. M. 2004 Contact problems and depth-sensing nanoindentation for frictionless and frictional boundary conditions. Int. J. Solids Struct. 41, 2479-2499. 
Bower A.F., Fleck N.A., Needleman A. and Ogbonna N., 1993 Indentation of a power law creeping solid. Proc. R. Soc. Lond. A 441, 97-124

Felder E., 2006. Analytical correlation of indentation experiments. Philos. Mag. 86, 5239.

Hernot X., Bartier O., Berkouche Y., El Abdi R., Mauvoisin G., 2006. Influence of penetration depth and mechanical properties on contact radius determination for spherical indentation. Int. J. Solids Struct. 43 (14-15), 4136-4153.

Hill, R., Storakers, B., Zdunek, A.B., 1989. A theoretical study of the Brinell hardness test. Proc. R. Soc. London A 423, 301-330.

Johnson, K.L.., 1970. The correlation of indentation experiments. J. Mech. Phys. Solids, 18 (1970), pp. 115-126

Larsson P-L. 2008. Similarity methods for analysing indentation contact problemsAdvantages and disadvantages. Journal of materials processing technology 202 15-21

Lee H., Lee, J.H. and Pharr G.M., 2005. A numerical approach to spherical indentation techniques for material properties evaluation. J. Mech. Phys. Solids 53, 2037-2069.

Love, A. E. H., Boussinesq's problem for rigid cone.1939, Q. J. Math. (Oxford), 10, 161-175.

Matthews, J.R., 1980. Indentation hardness and hot pressing. Acta Metallurgica 28, 311-318.

Meyer, E., 1908. Untersuchen über Härteprüfung und Härte. Zeitschrift des Vereins deutschen Ingenieure 52, 645-654.

Segedin, C.-M., 1957 The relation between load and penetration for a spherical punch. Mathematika, 4, 2, 156-161.

Shu, J.-Y. and Fleck, N.-A., 1998. Int. J. Solids Structures 35, 13, 1363-1383.

Sneddon, I.-N., 1965. The relation between load and penetration in the axisymmetric Boussinesq problem for a punch of arbitrary profile. Int. J. Eng. Sci. 3, 47-57

Storackers, B. and Larsson, P.-L., 1994. On Brinell and Boussinesq indentation of creeping solids. J. Mech Phys. Solids 42, 307-332.

Taljat, B., Zacharias, T., Kosel, T., 1998. New analytical procedure to determine stress-strain curve from spherical indentation data. International Journal of Solids and Structures 35 (33), 4411-4426. 